\title{
Concepciones de educadores en formación sobre la autorregulación del aprendizaje y la práctica profesional
}

Conceptions of educators in training on the self-regulation of learning and professional practice

García Montero Ivet*

ivet2010@gmail.com

Doris Castellanos Simons* doriscastellanoss@gmail.com

Universidad Pedagógica Nacional, México*

Centro de Investigación Transdisciplinar en Psicología,

Universidad Autónoma del Estado de Morelos*

DOI: 10.32654/CONCIENCIAEPG.3-1.2

Para referenciar este artículo: Ivet, G., \& Castellanos Simons, D. (2018). Concepciones de educadores en formación sobre la autorregulación del aprendizaje y la práctica profesional. REVISTA ConCiencia EPG, 3(1), 31-46. doi: https://doi.org/10.32654/ CONCIENCIAEPG.3-1.2
Fecha de recepción : 03 - 05-2018|Fecha de aceptación :26-06-2018

\section{Resumen}

$\checkmark$ 1 artículo presenta los avances de un proyecto más amplio que se desarrolla en una unidad de la Universidad Pedagógica Nacional, México, con 32 estudiantes de la Licenciatura en Intervención Educativa, con miras a fortalecer la calidad de los procesos de formación profesional y de aprendizaje desde una perspectiva integradora que tome en cuenta factores diversos. En particular, se reportan aquí resultados parciales de una etapa que tuvo como objetivos identificar sus concepciones sobre el proceso de autorregulación y el papel que tiene en la formación profesional, así como conocer sus representaciones acerca de su futura práctica educativa, las exigencias que la impactan y las necesidades formativas propias en el contexto sociocultural actual.

El estudio se enmarca en el modelo cualitativo; su diseño corresponde a la investigación acción participativa, cuya consecución posibilitó la participación activa de los estudiantes en las acciones de reflexión, problematización y de búsqueda de solución práctica de sus necesidades. Se emplearon grupos focales, la observación participante, cuestionarios y entrevistas. Los resultados muestran que los estudiantes poseen escasos conocimientos acerca de la autorregulación y de su papel en sus procesos formativos. Se concluye que la importancia de comprender la autorregulación es un proceso complejo, cuya formación se relaciona tanto con procesos psicológicos, como con factores socioculturales y educativos diversos que deben ser desarrollados para promover nuevas competencias de estructuración, contextualización y gestión de los saberes, que los estudiantes necesitan para transformar su realidad y transformarse a sí mismos en su trayecto académico.

Palabras clave: competencias, autorregulación, aprendizaje, educación superior 


\section{Summary}

7 he article presents the advances of a study carried out in the National Pedagogical University, in Morelos, Mexico, with 32 students of the Degree in Educational Intervention. It has among its essential objectives, to assess the competences for the self-regulation of the students' learning, identify and value their conceptions about this process and about the role that the same has in their professional formation, as well as recognize their representations about their future educational practice, the demands that impact it and their own educational needs in the current sociocultural context.

The research was carried out with the aim of strengthening the quality of the processes of professional training and learning from an integrative perspective that takes into account both the psychological dimension of learning and the didactic-pedagogical and socio-cultural aspects that affect professional training.

Theoretically, it relies on the contributions of the disciplines that explain the complexity of the processes that mediate the professional preparation of educators. The study is part of the qualitative model; its methodological design corresponds to the participatory action research, whose achievement allows the active participation of students in the problematization actions and practical solution of their needs.

Focus groups, participant observation and interviews are used as essential techniques. The preliminary results show that students have little knowledge about self-regulation and its role in their training processes and show signs of low levels of development in their self-regulatory skills. It is concluded that self-regulation is a complex process, whose training is related both to psychological processes, as well as to various socio-cultural and educational factors that must be developed to promote new structuring, contextualization and knowledge management competencies, which students need to transform their reality and transform themselves in their academic journey.

Key words: competencies, self-regulation, learning, higher education

\section{Introducción}

$\int$ 1 trabajo que se presenta muestra los avances de una investigación que se realiza en el contexto de la Universidad Pedagógica Nacional, en México, dirigida a fortalecer las competencias para la autorregulación del aprendizaje en estudiantes de la Licenciatura en Intervención Educativa.

El proyecto, en sentido más amplio, pretende generar una propuesta teóricometodológica para la comprensión de la autorregulación del aprendizaje y contribuir a la formación de aprendices autorregulados en sus contextos de práctica real, en este caso, encaminada a la formación profesional para ser interventores y educadores, en general.

El surgimiento de la investigación responde, puntualmente, a las necesidades formativas de los jóvenes universitarios, tomando como referentes los saberes profesionales que se plantean en el perfil del interventor educativo, así como del docente actual. 
De igual forma, se consideran las demandas a las que deben responder los estudiantes que se forman en educación superior relacionadas con las múltiples exigencias socioeconómicas que enfrenta la nación y con los procesos de cambio e incertidumbre que se han generado en el ámbito laboral.

Los jóvenes deben contar con saberes propios que les permitan enfrentar tales situaciones y aprender por sí mismos todo lo necesario para insertarse en el entorno sociolaboral.

Contar con instrumentos de autorregulación del aprendizaje, ser capaces de plantearse nuevas metas de aprendizaje y desarrollo, y de tomar decisiones acerca de sus formas de consecución, parece ser una opción que distingue las posibilidades de éxito ante tales exigencias.

En este sentido, se hace énfasis en el desarrollode aquellos mediadores psicológicos que permiten conducir $y$ fortalecer los procesos de aprendizaje; por ello, entre los fines del estudio se destaca la profundización en el análisis de las competencias para la autorregulación, en particular, y en línea con las propuestas teóricas y metodológicas de Rodríguez-Mena, García, Corral y Lago, 2004), la competencia de estructuración, de gestión y de contextualización del saber propio.

El estudio se sustenta en una visión teórica del proceso de autorregulación, basada en el Enfoque histórico-cultural, nacido con la obra de L. V. Vigotsky (1987) y continuado
Artículos Empíricos

por un grupo de autores que desarrollaron los principios esenciales del mismo.

De ese modo, se busca también una explicación a los procesos formativos de los jóvenes más allá de la perspectiva sociocognitiva (Zimerman, 2002, 2008) que ha tenido una fuerte presencia en las investigaciones actuales sobre la autorregulación, sobre la autorregulación en docentes y en maestros en formación (Aguilar, 2015; Brennan, 2016; Peeters, De Backer, Romer, Kindekens, Buffel, \& Lombaerts, 2014, Quintana, 2015, Ramírez, 2017), pero que a nuestro juicio, incorpora de manera limitada los determinantes socioculturales esenciales de los procesos de desarrollo profesional.

La concepción vigotskiana del desarrollo psicológico (Vigotsky, 1987) permite interpretar a la autorregulación como formación psicológica cualitativamente superior, como cualidad y meta del desarrollo psicológico humano.

El ser humano se apropia de los instrumentos culturales que le permiten transformar su mente $\mathrm{y}$, como consecuencia, desarrollar funciones psíquicas intrapersonales que le garantizan la autorregulación de sus aprendizajes y su propio desarrollo, pues se convierten en mediadores psicológicos que le proporcionan posibilidades ilimitadas de autodesarrollo para, a su vez, continuar apropiándose de la cultura.

En este trabajo, se asume la autorregulación desde la propuesta de Rodríguez-Mena, García, Corral y Lago (2004) que parte de los postulados del Enfoque Histórico Cultural, según la cual:

La autorregulación del aprendizaje implica un modo de aprender independiente y activo regido 
por objetivos y metas propios; supone el dominio y aplicación planificada y adaptable de recursos y procesos, referidos a estrategias metacognitivas, estrategias cognitivas, y procesos de dirección y control del esfuerzo, así como de componentes motivacionales, los que en su conjunto permiten resultados valiosos en los disímiles contextos en los que se inserta la "persona-que-aprende". Constituye un proceso complejo de carácter superior que se forma socialmente y se fortalece a lo largo del desarrollo de la personalidad de la persona-que-aprende (p. 15).

El desarrollo de la autorregulación involucra, desde esta visión, diversos procesos cognitivos, metacognitivos, motivacionales, afectivos y autorreferentes, lo que determina su carácter complejo (Labarrere, 1994, 2006). Entre los aspectos esenciales que determinan su conformación aparece el pensamiento verbal, dado el papel regulador que este asume frente a los motivos y comportamientos de las personas.

En relación con esta forma de pensamiento, aparecen las concepciones y representaciones que las personas construyen acerca de su entorno y de los fenómenos y procesos con los que se relaciona.

Precisamente, un eje interesante de análisis en el ámbito educativo ha sido, no sólo la reflexión acerca de cómo las representaciones sociales y las concepciones o teorías implícitas tienen un papel regulador en los procesos de aprendizaje y enseñanza, sino también sobre cómo las acciones educativas explícitas en un contexto esencial de formación social como la escuela, pueden conducir a la conformación de tales procesos psicosociales y su reestructuración (Pozo y Scheuer, 2011).

Según Pozo, Scheuer, Mateos y Pérez(2011), una coincidencia en muchos de los estudios realizados es que las teorías implícitas, como concepciones propias (se consideran en este trabajo ambos términos como sinónimos), subyacen las acciones y decisiones que toman los aprendices y maestros en el entorno escolar, sin que tengan pleno dominio de tales concepciones a nivel consciente.

Las concepciones y teorías implícitas se construyen como resultado de la exposición repetida a situaciones de aprendizaje culturalmente organizadas, ancladas en representaciones sociales que han trascendido por generaciones de docentes y aprendices, y dan sentido a las prácticas de enseñanzaaprendizaje en el contexto social de la escuela.

En ese sentido, ejercen su papel regulador en distintos niveles, algunas aparecen profundamente arraigadas en estructuras cognitivas de los aprendices y maestros y tienen carácter estable, otras están más ligadas a actuaciones que se marcan en los contextos y escenarios sociales de pertenencia, y pueden ser más flexibles y modificables.

Las ideas expuestas por Pozo y sus colaboradores (2011) son congruentes con la visión general, explicada por Vigotsky y sus seguidores (Leontiev, 1981; Talizina, 1988), en torno al papel regulador del pensamiento verbal y de los significados que las personas otorgan a los fenómenos, objetos, procesos y relaciones en los que están inmersos.

De acuerdo con Vigotsky, el ser humano se diferencia de los animales por su actividad de signación: crea y emplea símbolos que son 
algo así como señales convencionales de las que se sirve como instrumento para dominar la conducta ajena o propia (Vigotsky, 1987).

Los signos son introducidos por las personas en la situación psicológica y cumplen la función de autoestimulación, y en este contexto, el significado pasa a ser la dimensión semántica del procedimiento sígnico; es decir, el significado es quien posibilita al signo referirse al objeto.

Como es conocido, Vigotsky privilegió el estudio del lenguaje como sistema simbólico. Él encontró en la palabra la unidad psíquica básica para entender la relación entre pensamiento y lenguaje y entre las funciones comunicativa e intelectual de este último.

Los significados son los conceptos que definen los objetos, sus propiedades, nexos, leyes y acciones que el hombre realiza con ellos. Son descubiertos y generados en la práctica social conjunta desde lo intersubjetivo; y una vez interiorizados permiten la reflexión abstracta e intervienen, desde lo intrasubjetivo, en todas las operaciones que implican pensamiento consciente: la significación es un fenómeno inherente al pensamiento (Vigotsky, 2005).

A su vez, Leontiev (1981) apunta que no existe una coincidencia estricta entre la significación social (para la que reserva el término significado) y la significación en la conciencia individual, a la que denomina sentido personal.

Este último término alude a la relación que la significación tiene con los motivos de la actividad; por tanto, a diferencia de los significados que tienen existencia no-psicológica, objetiva, igual para todos los sujetos; el sentido personal solo existe en el plano psicológico: su función es hacer subjetiva la significación social.

Desde estas bases teóricas puede comprenderse la importancia que toman las significaciones, tanto las compartidas socialmente, como aquellas que permiten dar sentido personal a las actividades de las personas desde la perspectiva psicológica.

En ese sentido, la hipótesis implícita en el estudio que se presenta es que, tanto las representaciones que poseen los jóvenes acerca de la autorregulación, expresadas en concepciones apropiadas a partir de las influencias cultuales y sociales que los han impactado, como la resignificación y sentido que les han dado a la misma en sus actividades de aprendizaje, tienen un papel determinante en los motivos que expresan para ser aprendices autorregulados y en la formación y desarrollo de sus competencias para la autorregulación del aprendizaje.

Este papel puede ser favorable, si existe una adecuada comprensión y significación, o resultar negativa, si los significados construidos no son coherentes con los significados elaborados científicamente por la psicología educativa, lo que avala la necesidad de desarrollar procesos de autorregulación para lograr calidad en el aprendizaje.

Los supuestos antes mencionados son coherentes con los resultados de diversos estudios que abordan los procesos de autorregulación en contextos educativos (Fernández, Bernardo, Suárez, Cerezo, Núñez \& Rosario, 2015; 2013; García, 2012; García-Jiménez, 2015; KellerSchneider, 2014; Ramírez, 2017, Rosário, Pereira, 
Nunes et al., 2014; Sáiz-Manzanarez y Pérez, 2016; Tuckman, 2003).

En ellos se ha estacado el hecho de que las representaciones que intervienen en los procesos de autorregulación están relacionadas con la manera en que es concebido su propio significado, o con las concepciones apropiadas acerca de las acciones la planificación, anticipación, ejecución y control de las tareas de aprendizaje, y que igualmente, intervienen otras representaciones de carácter social vinculadas al aprendizaje mismo y la enseñanza, al rol del aprendiz y, en el caso de la formación de estudiantes universitarios, aquellas relacionadas con la formación profesional.

Todas ellas inciden en la importancia de la comprensión que se pueda tener de la autorregulación del aprendizaje, y en las valoraciones que los aprendices hacen de su importancia en su desempeño escolar y como futuros profesionales.

A partir de tales presupuestos, una de las direcciones de la investigación fue reconocer las representaciones y teorías implícitas de los aprendices vinculadas, a la autorregulación del aprendizaje, dado que el desarrollo y formación de sus competencias implicaría necesariamente tomar conciencia de su significado y otorgar sentido al proceso en el marco de su formación profesional.

De igual manera, podría también contemplarse la necesidad de reestructurar las concepciones apropiadas socioculturalmente que no son coherentes con la significación científica del proceso desde la perspectiva psicopedagógica.

En otras palabras, el análisis de las representaciones o concepciones de los aprendices permitiría convertir el desarrollo de la autorregulación en un motivo y meta propios que guíen de manera consciente la búsqueda de las competencias necesarias para llegar a ser un aprendiz autorregulado.

Por lo anteriormente expuesto, los objetivos del presente estudio fueron identificar las representaciones y concepciones de los estudiantes acerca de la autorregulación del aprendizaje y su papel en la formación profesional, así como sus percepciones acerca de su futura práctica educativa, las exigencias que la impactan y las necesidades formativas propias en el contexto sociocultural actual.

\section{Método}

Tipo de estudio: El estudio tiene sus bases metodológicas en la investigación cualitativa, por sus potencialidades en la comprensión de procesos psicopedagógicos complejos, como sucede con el estudio de la autorregulación del aprendizaje y su formación; de igual forma, por las posibilidades que brinda de estudiar estos fenómenos en un contexto real de práctica formativa y de proponer y valorar procesos de intervención psicopedagógica como parte de la investigación.

El enfoque metodológico cualitativo que se propone se materializa en un diseño investigación-acción participativa y crítica, sustentado en la interpretación y el análisis fenomenológico (Arnal, Del Rincón \& Latorre, 1992).

Diseño: El diseño de investigaciónacción participativa propuesto estuvo integrado por fases secuenciales de acción que involucraron: la planificación a partir de la problematización inicial, la identificación 
colaborativa de hechos, problemáticas y perspectivas subjetivas en torno a los mismos, el análisis participativo de la situación que viven en el proceso de formación contextualizado, propuesta e implementación del proceso de intervención y evaluación, para nuevamente abrir una fase de valoración de la problemática y su transformación.

En particular, la exploración de las concepciones de los estudiantes en torno a su futura práctica docente, sus exigencias y necesidades formativas propias, así como acerca de la autorregulación del aprendizaje y su papel en la formación profesional (reportados en este trabajo), se llevó a cabo durante las tres primeras etapas de problematización y diagnóstico inicial y profundo.

En todos los casos, las técnicas empleadas no sólo permitieron obtener información, sino que han constituido instrumentos mediadores para la reflexión y el diálogo con los participantes, quienes han sido colaboradores activos de la interpretación y análisis de los resultados tomando conciencia de los factores relacionados con el nivel de desarrollo de las competencias de autorregulación y de las necesidades formativas respecto a las mismas.

Participantes: Los participantes del estudio fueron estudiantes de la Generación 2015-2019 de la Licenciatura en Intervención Educativa en una de las sedes de la Universidad Pedagógica Nacional en Morelos.

El grupo inicial estuvo conformado por 32 estudiantes (10 hombres y 22 mujeres), con un rango de edad entre los 19 y 22 años.
Recolección de la información y análisis de los datos: Se emplearon los grupos focales, la autobiografía, la entrevista en profundidad, el diario de campo, así como dos cuestionarios: el primero, para la exploración de los datos generales sociodemográficos e historia escolar de los participantes y datos de su trayectoria escolar, y el segundo para el estudio de sus representaciones y concepciones respectivamente.

Para el procesamiento de la información obtenida con los cuestionarios, así como los grupos focales, reportados en este trabajo, se transcribieron íntegramente las participaciones de los estudiantes, y se empleó el análisis de contenido de sus respuestas, en la búsqueda de las categorías emergentes a un nivel descriptivo y también analítico e interpretativo.

\section{Resultados}

Características sociodemográficas y contexto cultural de los participantes: A partir del diagnóstico de los aspectos sociodemográficos, se sistematizaron datos sustanciales de la historia personal y escolar de los 32 jóvenes del grupo participante, que se resumen a continuación:

- La mayoría de los jóvenes (91.17 \%) convive con sus familias las que, además, se caracterizan por ser nucleares y extendidas, y se destacan por la presencia de ambos padres, evidenciando unidad familiar y posible estabilidad, existiendo un predominio de casos en los que se expresa la presencia del apoyo familiar económico y afectivo.

- Se dedican fundamentalmente a estudiar; así, 18 estudiantes declaran que esta es su actividad social exclusiva.

- La casi totalidad de los participantes mantiene vínculos de dependencia con relación 
a su familia y probablemente sus decisiones y comportamientos estén determinados por sus padres. La mayoría de ellos son solteros.

Sólo tres de las estudiantes tienen hijos, tres estudiantes han abandonado temporalmente sus estudios por embarazos

Así, con relación a otros datos socioeconómicos de interés, se pudo conocer que la mayoría de los estudiantes, provienen de familias con un nivel socioeconómico medio bajo.\}

Los resultados indican que los jóvenes tienen influencia directa de sus familias y dependen para realizar sus actividades de las mismas, dado que conviven con ellas.

Ello permite inferir que están expuestos de manera predominante a mecanismos reguladores, que son frecuentes en la vida sociofamiliar del contexto cultural, lo que podría ser un factor que incida de forma negativa en la formación de proceso de autorregulación de la personalidad.

El hecho de que los jóvenes estén, casi en su totalidad, dedicados a la actividad de estudio constituye un aspecto favorable para convertir la mejora de sus competencias de aprendizaje $\mathrm{y}$, en particular, las metas de desarrollo de la autorregulación del aprendizaje, en uno de los motivos jerárquicamente dominantes, sin que los mismos puedan entrar en conflictos con otras metas importantes en la etapa de desarrollo en que se encuentran tales como la formación de la familia propia y la búsqueda de empleo (Domínguez, 2005).
Trayectoria escolar previa de los estudiantes como posible antecedente de sus concepciones acerca de la autorregulación:

Losresultados delanálisis delas autobiografías, así como de las entrevistas a los jóvenes permitieron valorar aspectos relacionadas con su formación escolar que constituyen indudables antecedentes para su desarrollo como aprendices autorregulados, así como para la formación de representaciones en torno a este proceso y a aquellos relacionados, en sentido amplio, con el aprendizaje y la enseñanza. A continuación, se presenta una síntesis de los resultados encontrados.

-La casi totalidad de los alumnos cursaron sus estudios de nivel básico y medio superior en instituciones públicas (preescolar: $82 \%$, primaria: $94 \%$, secundaria: $91 \%$ y nivel medio superior: 94\%), lo que puede relacionarse con las propuestas del modelo educativo mexicano vigente hasta 2017, que, según Morán (2010) estuvo marcado por una tendencia tradicional y verbalista, que propició una docencia que fomenta pasividad, dependencia y conformismo en los estudiantes, lo que aún tiene efectos en el alumnado universitario.

-Los jóvenes manifestaron en sus respuestas vivencias negativas asociadas a la experiencias docentes autoritarias, reguladoras e inhibidoras de la autonomía y el desarrollo cognitivo. En particular, relataron experiencias en las que predominó la regulación externa de los procesos de aprendizaje por parte de los maestros, limitándoles a participar, cuestionar $\mathrm{u}$ ofrecer opiniones propias.

En este sentido, se identificaron 
manifestaciones de dependencia respecto a las decisiones de docentes para orientar su desempeño escolar, y prácticamente muy pocas referencias a experiencias que indiquen autonomía y expresión de acciones autorreguladoras.

En síntesis, emerge de sus respuestas la prevalencia de una experiencia académica previa centrada en objetivos de aprendizaje que destacan una orientación escolar vinculada a la reproducción y a la regulación sobre la base estímulos y recompensas externas.

Otros aspectos detectados a partir de las respuestas de los estudiantes al primer cuestionario de datos generales y sociodemográficos fueron:

-La manifestación de motivos dominantes de contenido social (relaciones de amistad y de pareja) que se superponen a las metas académicas en el contexto educativo; por lo general, refirieron que las experiencias más positivas y motivantes fueron aquellas vinculadas con hacer amigos, y conocer personas en el contexto escolar.

-El decrecimiento en los índices de rendimiento académico a lo largo de la trayectoria escolar, expresado en que disminuye el número de estudiantes que reportan un promedio de nueve o más en su formación previa

El análisis de las trayectorias de los participantes en el estudio permitió corroborar que la formación escolar precedente a la educación superior estuvo permeada por procesos psicopedagógicos reguladores y generadores de dependencia académica, conducidos por acciones docentes autoritarias, verbalistas y transmisivas.

Este tipo docencia, por lo general, promueve actitudes pasivas y receptivas, más que comportamientos autónomos y de gestión del propio aprendizaje. Los jóvenes describen muchas de sus experiencias como frustrantes y reconocen que fueron negativas y poco alentadoras para su desarrollo y desempeño escolar.

Puede inferirse que las representaciones en torno a la enseñanza y el aprendizaje que fueron construidas por ellos están marcadas por esta concepción, la que se refleja en el discurso predominante en los participantes acerca del papel de los docentes como aquellos que determinan sus objetivos y formas de aprendizaje.

En ningún caso, se hizo referencia a experiencias relacionadas con la formación para la autorregulación del aprendizaje o procesos relacionados con ella.

Representaciones y concepciones de los aprendices en torno a la autorregulación del aprendizaje y su importancia en la formación profesional: Uno de los objetivos fundamentales del estudio fue explorar las representaciones y concepciones de los estudiantes, que se expresan en gran medida en las ideas y conceptos que manejan relativos, específicamente, a la autorregulación del aprendizaje.

Esto facilita aproximarse a la comprensión que tienen del proceso y como consecuencia, valorar sus posibilidades para utilizar sus propias ideas en la regulación de sus comportamientos y el desarrollo de la propia autorregulación. En ese sentido, dicha exploración es imprescindible desde los principios planteados por Vigotsky (2005) en 
torno al papel autorregulador del pensamiento verbal.

Con relación al significado que para ellos tiene la autorregulación del aprendizaje, se constató un predominio de teorías implícitas y preconceptos, es decir, nociones espontáneas, sin sustento científico. Esto se relacionó asimismo con aspectos como:

-Presencia de expresiones de inseguridad con relación a sus ideas y elaboraciones inespecíficas o vagas respecto a cuestiones esenciales del concepto.

-En algunos casos, manifestación explícita de desconocimiento con relación a la autorregulación, aunque plantean interés por conocer su concepto. En otros casos la vaguedad se manifestó en el sentido tautológico: la autorregulación es entendida como regularse o está relacionada totalmente de forma confusa con otros significados.

Fue notorio que en los planteamientos de los estudiantes emergiera el carácter implícito de sus concepciones, con la expresión: "me suena que...", lo que indica la carencia de conocimientos específicos y el hecho de que están utilizando ideas estructuradas de forma espontánea.

No obstante, sí se establecieron, relaciones con aspectos relevantes tales como el hecho de considerar el papel de la persona en la planificación, la recuperación de los conocimientos aprendidos (transferencia), el uso de procesos mediadores tales como indagar, entre otros, lo que alude a la presencia de concepciones interpretativas acerca de lo que supone autorregularse.

Otras cuestiones significativas que emergen de las respuestas y que permiten identificar la presencia de concepciones sobre la autorregulación son:

-Identifican significados vinculados con la evaluación, es decir, la autorregulación entendida como proceso de evaluación o autoevaluación.

-Para algunos participantes la autorregulación significa repaso, refuerzo de lo aprendido, consolidación de manera individual de saberes apropiados en clases o con otras personas. En otras palabras, la autorregulación es entendida como estudio, probablemente hábito de estudiar que permite repetir y asegurar la consecución del modelo (estrategia de repetición).

-Comprensión de la autorregulación como autodidactismo.

En general, se encontró que solo un pequeño grupo de alumnos muestra expresiones con alusión explícita a contenidos relevantes de las competencias de autorregulación; aunque de manera aislada, sí logran destacar elementos definitorios de la autorregulación del aprendizaje que son pertinentes para la comprensión de este proceso.

A continuación, se ilustran algunos de los resultados más significativos a partir de la información aportada por los participantes. Cabe señalar que la información personal de los participantes fue omitida, apareciendo solamente los códigos asignados a cada uno.

En el ejemplo siguiente, se explicitan los significados de la autorregulación como proceso de control y de autoorganización, y se denotan indicios del reconocimiento de su papel en la mediación de los aprendizajes, como competencia de reestructuración de los 
saberes, cuando se refiere a "comprender lo que aprendemos".

Ante la pregunta: “¿Qué significa para ti la autorregulación del aprendizaje"?, un estudiante refiere: "Poder comprender lo que aprendemos en el tiempo y forma que nos lo proponemos; además de tener el control para querer aprender" (S 12)

En los fragmentos que se presentan más abajo, se expresa directamente en las palabras de los estudiantes, la comprensión de la autorregulación como proceso relacionado con el manejo de estrategias, se hace referencia a que el comportamiento autorregulado y estratégico se relaciona con la autonomía, con la capacidad de tomar decisiones propias respecto a los recursos de aprendizaje y al mejor modo de emplearlos en las situaciones de aprendizaje.

-Es la forma en que ayudas a que adquieras y logres construir el aprendizaje esperado. Con distintos métodos o técnicas. Tiene que ver con la forma de enseñar o ayudar con los conocimientos de otros (S 26).

-Es el hecho de poder aprender de una forma autónoma buscando nuestras propias estrategias de aprendizaje (S 27)

-Para mí es que el mismo sujeto que aprenda tenga la capacidad de medir y/o evaluar su aprendizaje, según un periodo o un determinado tiempo, de esa manera ser autónomo en su aprendizaje y desarrollo cognitivo (S 9)

Los ejemplos anteriores indican contenidos que están vinculados a las competencias de gestión del aprendizaje, tales como la toma de decisiones permanente durante las experiencias de aprendizaje acerca de sus fines y de las estrategias, métodos y técnicas para conseguirlo.

De igual forma, la capacidad de control es otro elemento que expresa la comprensión de la gestión, como se evidencia a continuación: (...) para mí la autorregulación del aprendizaje es como llevar un control del aprendizaje (S 9).

Se evidencia aquí la alusión directa respecto a la gestión como expresión de autorregulación, indicando un nivel mayor de comprensión de dicho proceso. Además, esta estudiante, que es una de las que muestra un mejor desempeño en clases, refiere otros aspectos sustanciales, tales como la orientación por interés propio, interpretable como la presencia de metas personales, la búsqueda de comprensión o estructuración como competencia autorreguladora y la búsqueda de nuevos saberes más allá de lo trabajado en clases, es decir, la comprensión de que la autorregulación supone expansión, actitud proactiva y constructiva.

Es cuando una persona gestiona su aprendizaje de manera individual para la comprensión o reforzamiento de algún tema en especial. Además de la búsqueda de información extracurricular o de interés propio (S 17)

En resumen, los resultados relacionados con el concepto que poseen acerca de la autorregulación indican que la mayoría de los estudiantes posee nociones implícitas o espontáneas, consideradas por Vigotsky en la explicación de la formación de los conceptos científicos como preconceptos (Vigotsky, 2005), lo que indica que carecen aún de conocimientos precisos para comprender de manera consciente y generalizada el concepto de 
autorregulación y aquellos relacionados con él.

Esto supone la falta de aspectos conscientes que permitan su manejo y transferencia a las experiencias del aprendizaje.

En pocas palabras es difícil proponerse una meta e involucrarse afectivamente con ella, en torno a algo que no se sabe lo que es.

Aquellos que poseen nociones más cercanas al concepto científico parece ser que cuentan con algunas experiencias previas relacionadas con la formación de la autorregulación, aunque no fueron reconocidas por ellos de manera directa en otras técnicas, cuestión que se pudo constatar, justamente en los grupos focales.

Finalmente, algunos aspectos significativos de las representaciones de los participantes acerca del papel de la autorregulación en su formación profesional emergieron desde sus planteamientos en los grupos focales.

A partir de las intervenciones de los estudiantes, se encontró que algunos jóvenes manifiestan concepciones muy vagas e imprecisas con relación a la importancia de la autorregulación del aprendizaje en su desarrollo profesional, lo que podría estar relacionado conla falta de concepciones cognitivas previas en torno a la autorregulación del aprendizaje y sus componentes. En otros casos, muestran una opinión más intuitiva, reconocen que existe relación e importancia, pero en este caso, la argumentación de sus ideas, como aspecto esencial de la competencia de estructuración, resulta insuficiente.

A pesar de las situaciones descritas y las dificultades mostradas, algunos estudiantes perciben una relación más estrecha entre la autorregulación del aprendizaje y su formación como educadores, e incluso llegan a anticipar que dicho proceso es parte ineludible de la formación profesional.

Por otra parte, como resultado de una reflexión surgida en el propio grupo focal, algunos alumnos llegaron a establecer relaciones entre la importancia de la autorregulación como vía mediadora para fortalecer su formación y su práctica profesional (donde educan a otros). Esta idea es relevante, porque justo la educación de los demás implica la necesidad de aprendizaje permanente y es una idea que subyace a la necesidad de convertirse en aprendices autorregulados.

\section{Conclusiones}

Los resultados del proceso de investigación-acción participativa hasta el momento mostrados revelan aspectos sustanciales que favorecen los primeros pasos en la construcción de un programa psicopedagógico para la formación y desarrollo de las competencias para la autorregulación del aprendizaje en los estudiantes de UPN.

Los primeros elementos relativos a la historia de aprendizajes de los participantes revelan la limitada presencia de antecedentes y de mediadores psicopedagógicos adecuados para el desarrollo de este proceso durante las etapas previas a la juventud y a su ingreso a la Universidad. Ello supone la manifestación de escasas experiencias previas de aprendizaje para convertirse en aprendices autorregulados, tanto desde el punto de vista conceptual, como procedimental y estratégico.

Los jóvenes requieren aprender a ser 
autorregulados, conocer los significados del proceso a partir de la apropiación de nuevos conceptos científicos y las reestructuraciones de sus nociones o preconceptos espontáneos por medio del diseño de situaciones de aprendizaje interventivas que favorezcan la apropiación de estos saberes, así como el desarrollo de sus instrumentos de aprendizaje para este fin.

Las primeras acciones puestas en marcha en torno a la reflexión compartida para sensibilizarlos en este sentido, propiciar la toma de conciencia, la búsqueda de información y la comprensión del proceso de autorregulación y sus componentes ya han comenzado a tener efectos positivos en los aprendices, que en momentos posteriores del estudio serán detallados.

Los mismos se han materializado en las tareas realizadas por ellos en la Universidad, por ejemplo, buscaron e incorporaron sustentos teóricos para interpretar los grupos focales tras la orientación realizada en clases, también manifestaron expresiones en su discurso en torno a las nuevas ideas acerca de la autorregulación del aprendizaje que habían incorporado, comenzaron a realizar interrogantes sobre temas relacionados con ella y comentarios de cómo estaban cambiando su manera de pensar.

Otra cuestión importante supone la reestructuración de sus vivencias, el fomento de nuevos estados afectivos asociados a las experiencias de autorregulación del aprendizaje y a su transformación como aprendices autorregulados; la reflexión permanente, la confrontación y el análisis crítico de las experiencias previas de aprendizaje debe ser una herramienta mediadora clave para el autodescubrimiento y el desarrollo de nuevas metas.

Justamente, la carencia de metas claras de aprendizaje, la falta de elaboración y de criterios viables para construirlas, cuestiones reconocidas por los participantes, han sido factores identificados en el diagnóstico determinantes en las dificultades para actuar como aprendices autorregulados.

En ese sentido, el diseño y puesta en marcha de estrategias para la construcción de metas y para el control y seguimiento de su consecución se establece como un presupuesto psicopedagógico clave en la formación de sus competencias.

La relación estrecha entre los procesos formativos y de evaluación o control permanente de las acciones psicopedagógicas de manera compartida en el marco del grupo, que deberá conformarse como comunidad de aprendizaje, son claves esenciales para la formación y desarrollo de las competencias de autorregulación

En esa perspectiva, la tesis esencial que se sostiene es que los procesos educativos pueden tener un papel determinante en la reestructuración y reconstrucción de representaciones implícitas, en tanto pueden ser mediadores claves para la transformación de la percepción social y las categorías sociales con las que operan las personas y los grupos.

De ese modo, el empleo de estrategias psicopedagógicas dirigidas a la reflexión colectiva y a la autorreflexión, así como de sensibilización acerca del origen de las representaciones propias y sus consecuencias en las relaciones sociales cotidianas podría permitir la concientización de las mismas, y generar nuevas metas de cambio de actitudes y conductas que han constituido el resultado de aquellas. 


\section{Referencias}

Aguilar, V. (2015). Hacia un modelo de autorregulación docente en educación superior. Tema: procesos de formación y actores de la educación. En Memorias Electrónicas del XIII Congreso Nacional de Investigación Educativa, Volumen 2, No1, 2015-2016, ISSN: 2007-7246.

Arnal, J., Del Rincón, D. \& Latorre, A. (1992). Investigación educativa. Fundamentos $y$ metodologías. Barcelona: Labor.

Brennan, H. L. (2016). Professional development effects on teachers' selfregulated learning. Doctoral Dissertation. Johns Hopkins University. Disponible en: https://jscholarship.library.jhu. edu/bitstream/handle/1774.2/40336/ S M I T H - D I S S E R T A T I O N - 2016. pdf? sequence $=1 \&$ isAllowed $=\mathrm{y}$

Domínguez, L. (2006). Psicología del desarrollo. Problemas, principios y categorías. México, Tamaulipas: Editorial Interamericana de Asesorías y Servicios S. A.

Eekelen, I. M., Boshuizen, H. P. A. \& Vermunt, J. D. (2005). Self-regulation in Higher Education Teacher Learning. Higher Education, 50, 447-471.

Fernández, E., Bernardo, A., Suárez, N.; Cerezo, R., Núñez, J. C., \& Rosário, P. (2013), Predicción del uso de estrategias de autorregulación en educación superior. Anales de Psicología, 29 (3), 865-875. Disponible en http://dx.doi.org/10.6018/ analesps.29.3.139341
García-Jiménez, E. (2015). La evaluación del aprendizaje: de la retroalimentación a la autorregulación. El papel de las tecnologías. Relieve, 21 (2), art. M2. DOI: http://dx.doi.org/10.7203/ relieve.21.2.7546

García, I. (2012). Estrategias para la autorregulación del aprendizaje en estudiantes de educación superior. Tesis doctoral. Centro de Investigación y Docencia del Estado de Morelos (CIDHEM), Cuernavaca, Morelos.

Keller- Schneider, M. (2014). Self-regulated learning in teacher education. The significance of individual resources and learning behaviour. Australian Journal of Educational \& Developmental Psychology, 14, 144-158. Diponible en https://www.newcastle.edu.au/_data/ assets/pdf_file/0005/139082/self-7schneider-2014.pdf

Labarrere, A. (1994). Pensamiento. Análisis y autorregulación en la actividad cognoscitiva de los alumnos. México: Ángeles Editores.

Labarrere, A. (1995). Autorregulación de la conducta y la personalidad. En Colectivo de autores. Adolescente cubano. Una aproximación al estudio de su personalidad (pp. 30-37) La Habana: Editorial Pueblo y Educación.

Labarrere, A. (2006). Aprendizaje, complejidad y desarrollo: agenda curricular para enseñar en los tiempos actuales. Revista de Psicología, 15(2), Pág. 65-76. doi:10.5354/07190581.2012 .17147 
Leontiev, A. N. (1981): Actividad, conciencia, personalidad. La Habana: Editorial Pueblo y Educación.

Morán, P. (2010). Hacia una evaluación cualitativa en el aula. Reto para comprender y transformar la docencia. En: La educación en México: continuidad, cambios y perspectivas. Comp. Leonor González. Estado de México: UAEM.

Peeters, J., DeBacker,F., Romer, V., Kindekens, A., Buffel, T. \& Lombaerts, K. (2014). The role of teachers' self-regulatory capacities in the implementation of selfregulated learning practices. Procedia: Social and Behavioral Sciences. Disponible en https://core.ac.uk/ download/pdf/81190820.pdf

Pozo, J. I. y Scheuer, N. (2011). ¿Qué cambia en las teorías implícitas sobre el aprendizaje y la enseñanza? Dimensiones y procesos del cambio representacional. En: Pozo, J.I., Scheuer, N., Pérez, M., Mateos, M., Martín, E. y De la Cruz, M. (2011). Nuevas formas de pensar la enseñanza y el aprendizaje. Las concepciones de profesores y alumnos. Barcelona: Graó.

Pozo, J. I., Scheuer, N., Mateos, M. y Pérez, M. (2011). Las teorías implícitas sobre el aprendizaje y la enseñanza. En: Pozo, J.I., Scheuer, N., Pérez, M., Mateos, M., Martín, E. y De la Cruz, M. (2011). Nuevas formas de pensar la enseñanza y el aprendizaje. Las concepciones de profesores y alumnos. Barcelona: Graó.
Quintana, M. C. (2015). El aprendizaje autorregulado en estudiantes de educación superior. Tema: procesos de formación y actores de la educación. En Memorias Electrónicas del XIII Congreso Nacional de Investigación Educativa 2.

Ramírez, M.C. (2017). Aprendizaje autorregulado: diferencias por campus de conocimiento en estudiantes universitarios. En Memorias Electrónicas del XIV Congreso Nacional de Investigación Educativa. San Luis Potosí, México del 20 al 24 de noviembre, año 3, No. 3, 2017-2018. Recuperado de: http://comie. org.mx/congreso/memoriaelectronica/v14

Rodríguez-Mena, M., García, I., Corral, R. \& Lago, C. (2004). Aprender en la empresa. Fundamentos sociopsicopedagógicos del Programa de Formación de Aprendices Autorregulados en Comunidades de Aprendizaje. La Habana: Prensa Latina.

Rodríguez-Mena, M. (2008). La naturaleza social de los aprendizajes. En G. Fariñas y L. Coelho (orgs.) Cadernos ECOS: Educação, Cultura e Desenvolvimento Humano. São Paulo: Terceira Margem Editora Didática Ltda.

Rosário, P.; Pereira, A.; Högemann, J.; Nunes, A. R.; Figueiredo, M.; Núñez, J.C. y otros (2014). Autorregulación del aprendizaje: una revisión sistemática en revistas de la base SciELO. Universitas Psychologica, 13 (2), 781-797.

Sáinz-Manzanarez, M. C. y Pérez, M. I. (2016). Autorregulación y mejora del 
autoconocimiento en resolución de problemas. En Psicología desde el Caribe, 33 (1). Disponible en http://dx.doi. org $/ 10.14482 /$ psdc.33.1.8076

Shunk, D. H. \& Zimmerman, B. J. (Eds.) (1998). Self-regulated learning: From Teaching to Self-reflective Practice. New York: Guilford Press

Talizina, N. (1988). Psicología de la enseñanza. Moscú: Editorial Progreso.

Tuckman, B. (2003). The effect of learning and motivation strategies training on college students'achievement. Journal of College Student Development, 4, 430-437.

Universidad Pedagógica Nacional (2002). Plan de estudios de la Licenciatura en intervención educativa. México: UPN. Recuperado de: http://www.lie.upn.mx/
Vigotsky, L. S. (1987). Historia del desarrollo de las funciones psíquicas superiores. La Habana: Editorial Científico Técnica.

Vigotsky, L. S. (2005). Pensamiento y Lenguaje. La Habana: Editorial Pueblo y Educación.

Zimmerman, B. (2002). Becoming selfregulated learned: An overview. Theory into Practice, 41, 64-72.

Zimmerman, B. (2008). Investigating Self-Regulation and Motivation: historical background, methodological developments, and future prospects. American Educational Research Journal, Thousand Oaks, 45 (1), 166183. 